\title{
Measurement of the neutron electric dipole moment via spin rotation in a non-centrosymmetric crystal
}

\author{
V.V. Fedorov ${ }^{\mathrm{a}}$, M. Jentschel ${ }^{\mathrm{b}}$, I.A. Kuznetsov ${ }^{\mathrm{a}}$, E.G. Lapin ${ }^{\mathrm{a}}$, \\ E. Lelièvre-Berna ${ }^{b}$, V. Nesvizhevsky ${ }^{b}, A$. Petoukhov $^{b}$, S.Yu. Semenikhin ${ }^{a}$,

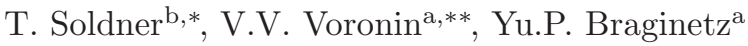 \\ ${ }^{a}$ Petersburg Nuclear Physics Institute, Gatchina, St.Petersburg, Russia \\ ${ }^{b}$ Institut Laue-Langevin, Grenoble, France
}

\begin{abstract}
We have measured the neutron electric dipole moment using spin rotation in a non-centrosymmetric crystal. Our result is $d_{\mathrm{n}}=\left(2.5 \pm 6.5^{\text {stat }} \pm 5.5^{\text {syst }}\right)$. $10^{-24} \mathrm{ecm}$. The dominating contribution to the systematic uncertainty is statistical in nature and will reduce with improved statistics. The statistical sensitivity can be increased to $2 \cdot 10^{-26} \mathrm{ecm}$ in 100 days data taking with an improved setup. We state technical requirements for a systematic uncertainty at the same level.

Keywords: electric dipole moment, CP violation, perfect crystal, neutron, diffraction, three-dimensional polarisation analysis

PACS: 14.20.Dh, 61.05.fm, 04.80.Cc
\end{abstract}

\section{Introduction}

Electric dipole moments (EDMs) of elementary particles belong to the most sensitive probes for CP violation beyond the Standard Model of Particle Physics [1]. Constraining or detecting EDMs of different systems allows to gather experimental information about models for new physics that is complementary to high energy physics data.

For the neutron EDM (nEDM), the most sensitive results [2, 3] were obtained using ultracold neutrons and Ramsey's resonance method. See [4] for a recent review of measurements using free neutrons. Measurements using the interaction of neutrons with the atomic electric field in absorbing matter were pioneered by Shull and Nathans [5]. Abov and colleagues [6] first discussed a spindependent term in the scattering amplitude for neutrons in non-centrosymmetric non-absorptive crystals. This term is caused by the interference of nuclear and

*soldner@ill.fr. Present address: Physik-Department E18, Technische Universität München, Garching, Germany.

**vvv@pnpi.spb.ru.

Preprint submitted to Elsevier

September 19, 2018 
spin-orbit structure amplitudes. Forte 7] proposed to search for nEDM related spin rotation in non-centrosymmetric crystals due to such interference. In [8] it was pointed out that such interference leads to a constant interplanar electric field affecting the neutron during all time of its passage through the crystal. This field was measured first in 9]. The interference of nuclear and spin-orbit structure amplitudes was tested by Forte and Zeyen [10] by spin rotation in a non-centrosymmetric crystal.

Here we present the first measurement of the nEDM based on an improved version of this method. Preliminary results and a detailed description of our method with references to earlier work have been published in conference proceedings [11, 12].

The statistical sensitivity of any experiment to measure the nEDM is determined by the product $E \tau \sqrt{N}$, where $E$ is the value of the electric field, $\tau$ the duration of the neutron's interaction with the field and $N$ the number of counted neutrons. New projects to measure the nEDM with UCNs aim to increase the UCN density and thus $N$ by orders of magnitude and exploit in some cases the higher electric field obtainable in liquid helium compared to vacuum [13, 14]. In contrast, experiments with non-centrosymmetric crystals exploit the interplanar electric field. For quartz, this field was measured to be $E \approx 2 \cdot 10^{8} \mathrm{~V} / \mathrm{cm}[9]$, several orders of magnitude higher than the electric field achievable in vacuum or liquid helium. Furthermore, the statistical sensitivity of the method profits from the higher flux of the used cold neutrons, compared to UCNs available today. These factors compensate the shorter interaction time of the neutrons with the electric field, ultimately limited by the absorption in the crystal. In [15] we have demonstrated that a statistical sensitivity of $\sim 6 \cdot 10^{-25} \mathrm{ecm} /$ day can be obtained, comparable to the most sensitive published UCN experiments [2, 3].

The results in [15] were obtained in Laue geometry that is limited by systematics [16]. Here we exploit the Bragg geometry first proposed by Forte [7]. See 11] for a detailed comparison of the two schemes.

\section{Method}

We consider spin rotation for neutrons close to the Bragg condition for the crystallographic plane $\boldsymbol{g}$ in a non-centrosymmetric crystal. These neutrons are exposed to an electric field $\boldsymbol{E}=\boldsymbol{E}_{\boldsymbol{g}} \cdot a$ where $\boldsymbol{E}_{\boldsymbol{g}}$ is the interplanar electric field for plane $\boldsymbol{g}$ and $a$ describes the deviation of the neutron from the exact Bragg condition (see 11] for details). A nonzero nEDM $d_{\mathrm{n}}$ results in neutron spin rotation by the angle

$$
\varphi_{\mathrm{EDM}}=\frac{2 E d_{\mathrm{n}} L}{\hbar v_{\perp}},
$$

where $L$ is the length of the crystal and $v_{\perp}$ the component of the neutron velocity perpendicular to the crystallographic plane $\left(L / v_{\perp}\right.$ is the time the neutron interacts with the field). By changing the deviation from the exact Bragg condition, $a$, value and even sign of the effective electric field and the resulting spin rotation $\varphi_{\mathrm{EDM}}$ can be selected. 
On the other hand, the electric field causes a Schwinger magnetic field in the rest frame of the neutron, $\boldsymbol{H}_{\mathrm{S}}=[\boldsymbol{E} \times \boldsymbol{v}] / c$, resulting in the spin rotation angle

$$
\varphi_{\mathrm{S}}=\frac{2 \mu_{\mathrm{n}} H_{\mathrm{S}} L}{\hbar v_{\perp}}=\frac{2 E \mu_{\mathrm{n}} L v_{\|}}{c \hbar v_{\perp}}
$$

where $\mu_{\mathrm{n}}$ is the neutron magnetic moment and $v_{\|}$the neutron velocity component parallel to the crystallographic plane.

As $\boldsymbol{E}$ and $\boldsymbol{H}_{\mathrm{S}}$ are perpendicular to each other, $\varphi_{\mathrm{EDM}}$ and $\varphi_{\mathrm{S}}$ can be separated by three-dimensional (3D) polarisation analysis. Furthermore, $\varphi_{\mathrm{S}}$ vanishes for Bragg angles of $\pi / 2\left(v_{\|}=0\right.$ in Eq. (2) $)$. This is used to suppress the effect due to Schwinger interaction: The crystal is aligned such that the interplanar electric field is parallel to the central neutron velocity, defining the $Z$ direction of a coordinate system. The incident neutron polarisation is aligned in $X$ (or $Y$ ) direction. $\varphi_{\text {EDM }}$ is measured in the $X-Y$ plane. A residual Schwinger magnetic field (for neutron trajectories deviating from the $Z$ direction or in case of a slight misalignment of the crystal) has its largest component in the $X-Y$ plane, thus creating a polarisation component in $Z$ direction. Thus, $\varphi_{\mathrm{S}}$ can be derived from the $Z$ component of the outgoing polarisation vector. In summary, we measure $\varphi_{\mathrm{EDM}}$ from the component $P_{X Y}$ of the polarisation tensor and the residual Schwinger effect from the components $P_{X Z}$ and $P_{Y Z} \cdot P_{Y X}, P_{Z X}$ and $P_{Z Y}$ serve for control purposes.

In first order, the difference of the polarisation tensors for positive and negative effective electric fields is:

$$
\Delta \boldsymbol{P}=g_{\mathrm{n}} \tau_{0}\left(\begin{array}{ccc}
0 & -\left(H^{z} \frac{\Delta \tau}{\tau_{0}}+H_{\mathrm{EDM}}\right) & \left(H^{y} \frac{\Delta \tau}{\tau_{0}}+H_{\mathrm{S}}^{y}\right) \\
\left(H^{z} \frac{\Delta \tau}{\tau_{0}}+H_{\mathrm{EDM}}\right) & 0 & -\left(H^{x} \frac{\Delta \tau}{\tau_{0}}+H_{\mathrm{S}}^{x}\right) \\
-\left(H^{y} \frac{\Delta \tau}{\tau_{0}}+H_{\mathrm{S}}^{y}\right) & \left(H^{x} \frac{\Delta \tau}{\tau_{0}}+H_{\mathrm{S}}^{x}\right) & 0
\end{array}\right)
$$

where $\tau_{0}=\left(\tau_{+}+\tau_{-}\right) / 2, \Delta \tau=\left(\tau_{+}-\tau_{-}\right) / 2$, and $\tau_{+}$and $\tau_{-}$are the times the neutrons stay in the crystal for the positive and the negative electric field, respectively (the neutron velocity is slightly different for $\pm a$ ). $H^{i}$ are the components of the residual magnetic field and $H_{\mathrm{S}}^{i}$ the components of the Schwinger magnetic field $\boldsymbol{H}_{\mathrm{S}} . g_{n}=2 \mu_{\mathrm{n}} / \hbar=1.8 \cdot 10^{4} \mathrm{G}^{-1} \mathrm{~S}^{-1}$ is the neutron gyromagnetic ratio, $H_{\mathrm{EDM}}=E d_{\mathrm{n}} / \mu_{\mathrm{n}}$ the effective magnetic field corresponding to the electric field $E$. For $E=1 \cdot 10^{8} \mathrm{~V} / \mathrm{cm}$ and $d_{n}=10^{-26} \mathrm{ecm}, H_{\mathrm{EDM}}=1.7 \cdot 10^{-7} \mathrm{G}$.

\section{Experimental setup and procedure}

The experiment was carried out at the cold neutron beam facility PF1B [17] of the Institut Laue-Langevin. A scheme of the setup is shown in Fig. 11 Neutrons were wavelength-preselected by a pyrolytic graphite monochromator (adjusted to $4.91 \AA$, resolution about $1 \%$ ) and spin polarised to about $98 \%$ by a super mirror polariser. A resonance spin flipper permitted to flip the neutron polarisation. Cryopad [18, 19] was used for 3D polarisation analysis. 


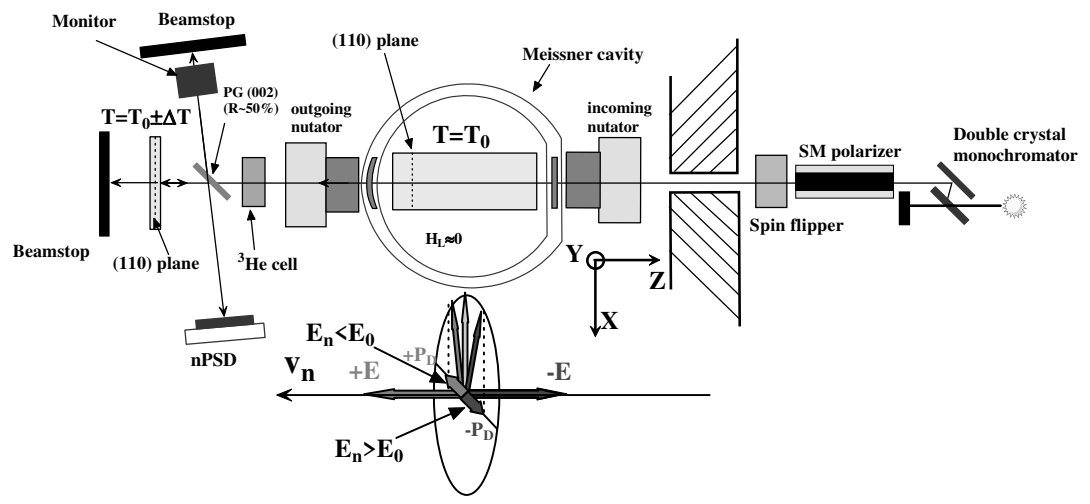

Figure 1: Scheme of the experiment. The neutrons come from the right. See Section 3 for details.

The direction of the incident beam polarisation was oriented and the measured projection of the outgoing polarisation vector selected by nutators (in the $X-Y$ plane) and precession coils (inside the Meissner screen, for the $Z$ component). The polarisation of the transmitted beam was analysed by a ${ }^{3} \mathrm{He}$ spin filter cell [20] (measured polarisation value of the unperturbed beam between $75 \%$ and $87 \%$, depending on the ${ }^{3} \mathrm{He}$ cell). The currents in the precession coils were optimised experimentally for the wavelength of the used neutrons.

We used the (110) reflection of a perfect quartz crystal of $14 \mathrm{~cm}$ length. The effective angular mosaic spread of the crystal was $\omega_{\mathrm{m}} \sim 1$ ". The neutrons with a well-defined deviation from the Bragg condition for this crystal were selected behind the ${ }^{3} \mathrm{He}$ spin filter cell by back-reflection at a second quartz crystal oriented parallel to the first one and at a slightly different temperature. A shift of the neutrons by one Bragg width $\left(\Delta \lambda_{B} / \lambda \approx 10^{-5}\right.$ for the (110) plane of quartz) corresponds to a temperature difference $\Delta T \approx \pm 1 \mathrm{~K}$ (linear coefficient of thermal expansion for quartz $\xi=\Delta L / L \approx 10^{-5} / \mathrm{K}$ ). Note that the absolute temperature of the crystals is not important. The crystals were temperature stabilised in individual housings connected by a common water circuit. The temperature of the second crystal was varied by Peltier elements.

The back-reflected neutrons were separated from the beam by a pyrolytic graphite monochromator (PG (002)) with a reflectivity of about $50 \%$ and directed to a position sensitive detector (nPSD). The nPSD allowed us to analyse data depending on the deviation of the neutron's path from the $Z$ direction: Larger deviations correspond to larger $v_{\|}$and thus $\varphi_{\mathrm{S}}$. This monochromator unavoidably reflects about $50 \%$ of the neutrons after the spin filter, before the second quartz crystal. These neutrons were used to monitor intensity and polarisation.

During installation, the crystals were pre-aligned optically using an autocollimator. Final alignment was performed with neutrons, allowing for the possibility of slightly different lattice constants of the crystals at the same temperature: 
The intensity of the neutron beam backscattered by the second crystal has a minimum if the Bragg condition of the second crystal (varied by the temperature difference) corresponds to the Bragg condition of the first one. If the crystal planes of both crystals are parallel, this minimum has the smallest width. The alignment with the minimum width was found by scanning the temperature difference for different angles (in $X$ and $Y$ direction) of the second crystal. Data show that the lattice constants were equal for $\Delta T=-0.8 \mathrm{~K}$ [11], which can be attributed to slightly different impurities of the two crystals. From previous measurements [21] we selected the working points with maximal electric field magnitude $\Delta T_{-}=-2.0 \mathrm{~K}$ and $\Delta T_{+}=+0.4 \mathrm{~K}$.

The different measured combinations of electric field direction and components of the polarisation tensor are $P_{i j \pm}$, where the index \pm refers to the temperature difference of the crystals $\Delta T_{ \pm}$. The indices $i$ and $j$ indicate the directions of the incident and outgoing neutron polarisation selected by Cryopad. Each component $P_{i j \pm}$ was measured for $40 \mathrm{~s}$ by flipping the incident neutron polarisation every $1-4 \mathrm{~s}$ with the resonance flipper (states $\uparrow$ off, $\downarrow$ on), in a drift-compensating scheme $\uparrow \downarrow \downarrow \uparrow \downarrow \uparrow \uparrow \downarrow$. The different states $P_{i j \pm}$ were measured in drift-compensating cycles, one for the EDM effect, $P_{X Y-}, P_{X Y+}, P_{X Y+}$, $P_{X Y_{-}}, P_{Y X_{-}}, P_{Y X_{+}}, P_{Y X_{+}}, P_{Y X_{-}}$, and one for the Schwinger effect, $P_{X Z_{-}}$, $P_{Y Z-}, P_{X Z+}, P_{Y Z+}, P_{Y Z+}, P_{X Z+}, P_{Y Z-}, P_{X Z-}$. EDM data were taken $2 / 3$ of the total time, Schwinger data due to the smaller required statistics only $1 / 3$. The dominating drift originated from the depolarisation of the ${ }^{3} \mathrm{He}$ cells with time (time constants large compared to a single cycle).

\section{Results}

From the measured data we determined the elements of the difference of the polarisation matrices, $\Delta P_{i j}=1 / 2\left(P_{i j+}-P_{i j-}\right)$. The Schwinger data showed the expected linear variation over the nPSD: neglecting the spatial extension of the beam, the deviation of a neutron from the centre of the nPSD is proportional to $v_{\|}$. Fig. 2 shows a linear fit to the data $\Delta P_{Z Y}$. This allows us to derive the electric field acting on the neutrons:

$$
E_{\exp }=(0.7 \pm 0.1) \cdot 10^{8} \mathrm{~V} / \mathrm{cm} \text {. }
$$

This value is consistent with our preliminary result obtained for the Bragg angle $\theta_{B}=86^{\circ}$ 21].

Fig. 3 (top) shows an example for the spatial dependence of the matrix element $\Delta P_{Y X}$ sensitive to the nEDM. The summary of all accumulated data for the $\mathrm{nEDM}$ is presented in Fig. 3 (bottom). The average value for the $\mathrm{nEDM}$ spin rotation is

$$
\varphi_{\mathrm{EDM}}=(0.9 \pm 2.3) \cdot 10^{-4} \mathrm{rad},
$$

corresponding to the $\mathrm{nEDM}$ (see Eq. (11) $) d_{\mathrm{n}}=(2.5 \pm 6.5) \cdot 10^{-24} \mathrm{ecm}$.

The slope of the spatial dependencies of $\Delta P_{Y X}$ and $\Delta P_{X Y}$ is related to the Schwinger effect. It was found to be consistent with zero and used to estimate a related systematic uncertainty of $\sigma d_{\mathrm{n}, \mathrm{S}}<5 \cdot 10^{-24} \mathrm{ecm}$. Note that 


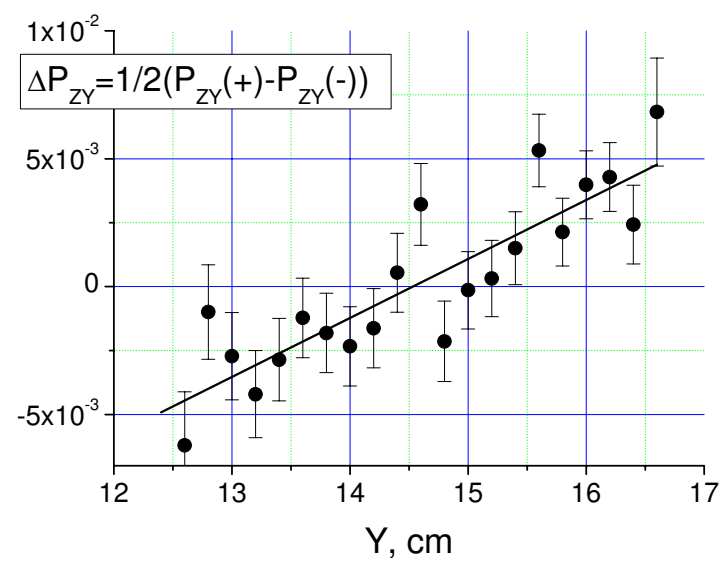

Figure 2: Measured dependence of $\Delta P_{Z Y}$ on the spatial coordinate $Y$ of the nPSD.

this uncertainty is smaller than the statistical uncertainty of the nEDM taken from the same data and would reduce with better statistics. It includes the error caused by imperfect angular accuracy of the 3D polarisation analysis (if the analysed $Z$ component is not perpendicular to the $X-Y$ plane, the corresponding projection of the Schwinger effect would contribute to $\Delta P_{X Y}$ and $\left.\Delta P_{Y X}\right)$.

The residual magnetic field inside Cryopad was $H \sim(1-2) \mathrm{mG}$. The reversal of the electric field leads to a small change of the neutron wavelength and thus to a difference of the time the neutron stays inside Cryopad. This time difference was measured directly: $\Delta \tau / \tau<5 \cdot 10^{-4}$. The combination of the residual magnetic field and the time difference results in a systematic uncertainty of $\sigma d_{\mathrm{n}, H}<4.5 \cdot 10^{-25} e \mathrm{~cm}$.

The time stability of the experiment was controlled by the beam monitor, which registered neutrons behind Cryopad without interaction with the second quartz crystal. The average electric field acting on these neutrons was about zero (taking into account the wavelength distribution in the neutron beam, it was $\sim 10^{3}$ times less than the field for the neutrons that would be selected by the second quartz crystal). The difference in polarisation measured by the beam monitor between the two working points, $\Delta P<2 \cdot 10^{-5}$, can be used to limit a possible systematic effect due to beam fluctuations in time, $\sigma d_{\mathrm{n}, \text { time }}<$ $10^{-24}$ ecm.

We have observed a spatial shift of the neutron beam at the nPSD correlated with the working point $\Delta T_{ \pm}$(see Fig. 4 ) , caused by an imperfect alignment of the two quartz crystals, and a spatial dependence of the polarisation (see Fig. 4b), probably due to the cylindrical shape of the outgoing window of Cryopad and the presence of a residual magnetic field. The combination of both effects causes a systematic error of $\Delta P_{\mathrm{S}}=1 / 2 \Delta Y_{\mathrm{S}} \delta P / \delta Y_{\mathrm{s}}$. For the final crystal alignment $\Delta Y_{\mathrm{S}}=0.03 \mathrm{~cm}$ this corresponds to $\Delta P_{\mathrm{S}}=4 \cdot 10^{-5}$ and $\sigma d_{\mathrm{n}, \mathrm{s}}=2 \cdot 10^{-24} e \mathrm{~cm}$.

Spin rotation caused by the weak interaction cancels for the two crystal 

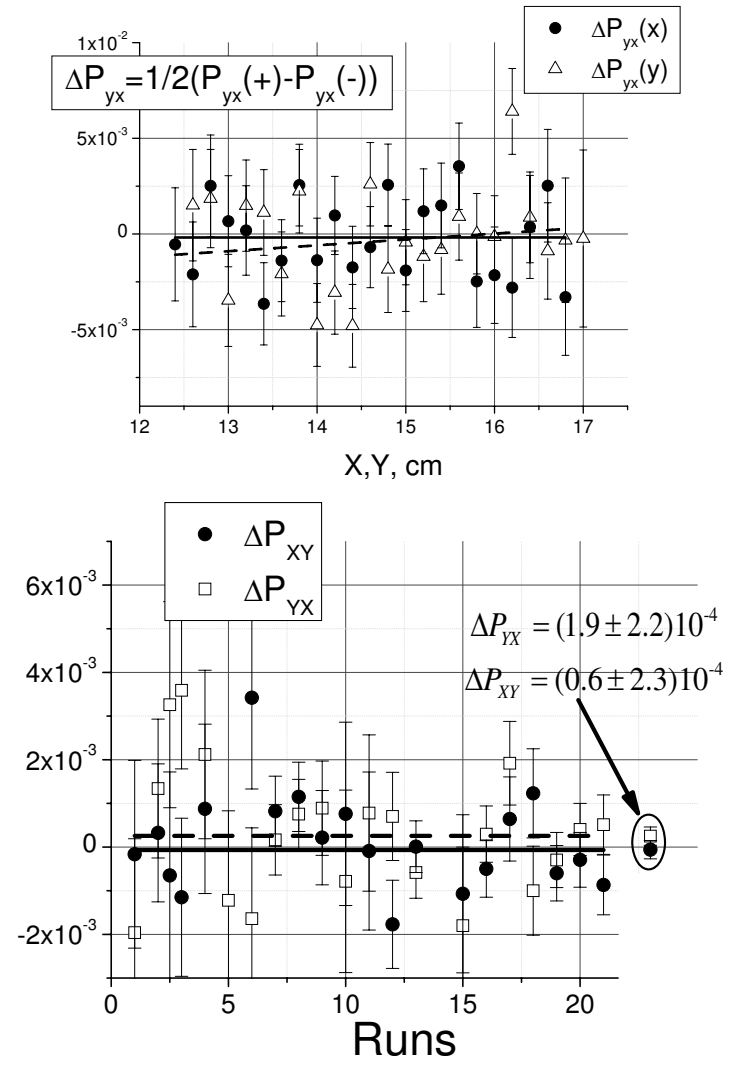

Figure 3: Top: Measured dependences of $\triangle P_{Y X}$ on the spatial coordinates $X$ and $Y$ of the nPSD. Bottom: Results of the different measurements of $\Delta P_{X Y}$ and $\Delta P_{Y X}$, used to derive the nEDM.

temperatures in first order. The cancellation is incomplete because of a cross term of weak and Schwinger rotation, but the uncompensated part is below $10^{-4}$ of the weak spin rotation and can be neglected.

Summing these independent systematic errors we obtain the final result

$$
d_{\mathrm{n}}=\left(2.5 \pm 6.5^{\mathrm{stat}} \pm 5.5^{\mathrm{syst}}\right) \cdot 10^{-24} e \mathrm{~cm} .
$$

Note that the systematic error is mainly statistical in nature. This result is about two orders of magnitude less precise than present experiments using UCNs and Ramsey's resonance method.

\section{Outlook}

The statistical sensitivity of the experiment described above was 1.6 . $10^{-23} \mathrm{ecm} /$ day. In [11] we have shown that the sensitivity can be improved by 


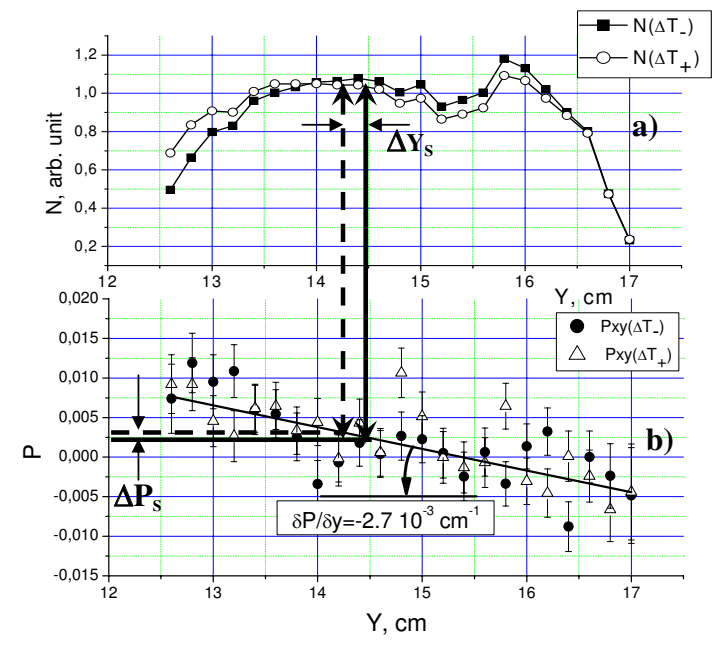

Figure 4: Spatial distribution of the registered neutron intensity a) and measured polarisation b) for the two working points $\Delta T_{ \pm}$.

Table 1: Experimental parameters for a systematic uncertainty of $\sigma d_{\mathrm{n}} \sim 10^{-26} \mathrm{ecm}$.

\begin{tabular}{|c|c|}
\hline Parameter & Value \\
\hline Interplanar distance variation $\Delta d / d$ & $<10^{-5}$ \\
\hline \multicolumn{2}{|l|}{ Residual magnetic field } \\
\hline \begin{tabular}{l|l} 
& Value \\
\end{tabular} & $\sim 10^{-4} \mathrm{G}$ \\
\hline Time stability & $\sim 10^{-5} \mathrm{G} / \mathrm{h}$ \\
\hline Accuracy of 3D polarisation analysis & $\sim 10^{-3} \mathrm{rad}$ \\
\hline Angular crystals alignment & $\sim 0.01^{\circ}$ \\
\hline Precision of temperature control & $\sim 0.01 \mathrm{~K}$ \\
\hline Flatness of Cryopad windows & $\sim 10^{-4} \mathrm{rad}$ \\
\hline
\end{tabular}

a factor 65 to about $2.5 \cdot 10^{-25} \mathrm{ecm} /$ day, essentially by increasing the divergence acceptance of the installation, the beam size, and the crystal length. This improved sensitivity is comparable to state-of-the-art nEDM measurements using UCNs and permits a statistical uncertainty at the $10^{-26} \mathrm{ecm}$ level in a beam time of 100 days. A further improvement may be possible by using crystals with higher interplanar electric fields. However, these crystals are presently not available in the required quality and size.

In Table 1 we list the experimental parameters that are required for an adequate systematic uncertainty. The values are achievable. Note that a variation of the interplanar distance in the crystal of the order of the Bragg width or larger does not simulate an nEDM but reduces the magnitude of the effective electric field.

We conclude that neutron spin rotation in quartz in Bragg geometry permits to measure the nEDM with a precision comparable to existing UCN ex- 
periments but using a completely different method. Efforts are now being made

to produce larger crystals and a version of Cryopad optimised for that kind of measurements.

Acknowledgements. The authors would like to thank the personnel of the ILL reactor (Grenoble, France) for technical assistance in the experiment. This work was supported by RFBR (grants No 09-02-00446).

\section{References}

[1] M. Pospelov and A. Ritz, Annals of Physics 318 (2005) 119.

[2] C.A. Baker, D.D. Doyle, P. Geltenbort, et al., Phys. Rev. Lett. 97 (2006) 131801.

[3] I.S. Altarev, Yu.V. Borisov, N.V. Borovikova, et al., Yad. Fiz. 59 (1996) 1204.

[4] S.K. Lamoreaux and R. Golub, J. Phys. G 36 (2009) 104002.

[5] C.G. Shull and R. Nathans, Phys. Rev. Lett. 19 (1967) 384.

[6] Yu.G. Abov, A.D. Gulko, and P.A. Krupchitsky, Polarized Slow Neutrons, (Atomizdat, Moscow, 1966) 256p. (in Russian).

[7] M. Forte, J. Phys. G 9 (1983) 745.

[8] V.L. Alekseev, E.G. Lapin, E.K. Leushkin, et al., JETP 67 (1988) 1727.

[9] V.L. Alexeev, V.V. Fedorov, E.G. Lapin, et al., Nucl. Instr. and Meth. A 284 (1989) 181; JETP 69 (1989) 1083.

[10] M. Forte and C.M.E. Zeyen, Nucl. Instr. and Meth. A 284 (1989) 147.

[11] V.V. Fedorov, M. Jentschel, I.A. Kuznetsov, et al., Nucl. Instr. and Meth. A 611 (2009) 124.

[12] V.V. Fedorov, M. Jentschel, I.A. Kuznetsov, et al., Nucl. Phys. A 827 (2009) 538.

[13] M.G.D. van der Grinten, Nucl. Instr. and Meth. A 611 (2009) 129.

[14] I. Altarev, G. Ban, G. Bison, et al., Nucl. Instr. and Meth. A 611 (2009) 133.

[15] V.V. Fedorov, E.G. Lapin, E. Lelièvre-Berna, et al., Nucl. Instr. Meth. B 227 (2005) 11.

[16] V.V. Fedorov, E.G. Lapin, S.Y.Semenikhin, et al., Int. Journ. Mod. Phys. A 23 (2008) 1435. 
[17] H. Abele, D. Dubbers, H. Häse, et al., Nucl. Instr. and Meth. A 562 (2006) 407.

[18] E. Lelièvre-Berna, P.J. Brown, F. Tasset, et al., Physica B 397 (2007) 120.

[19] E. Lelièvre-Berna, Novel polarized neutron tools, in I. Anderson and B. Guerard (eds.), Advances in Neutron Scattering Instrumentation, Proceedings of SPIE 4785 (2002), ISBN 0-8194-4552-5, pp. 112-125.

[20] A.K. Petoukhov, K.H. Andersen, D. Jullien, et al., Physica B 385 (2006) 1146.

[21] V.V. Fedorov, I.A. Kuznetsov, E.G. Lapin, et al., Nucl. Instr. and Meth. B 252 (2006) 131. 